\title{
Wilhelm Ehrhardt and an evaluation of his amphibians and reptiles held in the Herpetological Collection of the Museum für Naturkunde, Berlin
}

\author{
Alexander Gutsche*, ${ }^{*}$, Axel Kwet ${ }^{2}$, Christoph Kucharzewski ${ }^{3}$, Rodrigo Lingnau ${ }^{4}$ and Rainer Günther ${ }^{5}$ \\ 1 Humboldt-Universität zu Berlin, Department of Biology, Sensory Biology, Invalidenstrasse 43, 10115 Berlin, Germany \\ 2 Staatliches Museum für Naturkunde Stuttgart, Department of Zoology, Herpetology, Rosenstein 1, 70191 Stuttgart, Germany \\ 3 Bernauer Strasse 35 A, 13507 Berlin, Germany \\ 4 Museu de Ciências e Tecnologia - PUCRS, Laboratória de Herpetologia, Av. Ipiranga 6681, CEP 90619-900, Porto Alegre, \\ RS, Brasil \\ 5 Museum für Naturkunde der Humboldt-Universität zu Berlin, Invalidenstrasse 43, 10115 Berlin, Germany
}

Received 30 August 2006, accepted 4 November 2006

Published online 16 March 2007

With 1 figure

Key words: Wilhelm Ehrhardt, biography, historical collections, Zoologisches Museum Berlin, biodiversity, Brazilian Herpetofauna.

\begin{abstract}
Wilhelm Ehrhardt was a Guyana-born German animal collector and an excellent taxidermist. He operated in Brazil from 1897 until about 1935 and supplied several museums and scientific institutions with important items of various animal groups. The herpetological collections of W. Ehrhardt, stored in the Museum für Naturkunde, Berlin (ZMB) currently include 2416 specimens of 128 species, representing most of the native Brazilian groups of amphibians and reptiles. About $70 \%$ of the material was not determined and catalogued before, and all Ehrhardt specimens have been determined or re-examined, respectively. Here we present for the first time biographical data on W. Ehrhardt, and provide a complete catalogue of his herpetological collections held in the Museum für Naturkunde, Berlin. When necessary, brief remarks were given on the condition of the specimens, their taxonomy, systematics, and localities.
\end{abstract}

\section{Introduction}

Wilhelm Ehrhardt was an enthusiastic traveller, and he operated as professional animal collector and taxidermist in Brazil around the early $20^{\text {th }}$ century. Ehrhardt collected thousands of specimens of various animal groups, among them some new and well known amphibian, fish, and mammal species. For many years, he supplied several museums and scientific institutions, such as the British Museum of Natural History London and the Zoologisches Museum München, with important and well-labelled zoological material, that mostly was and often still is in excellent condition. More than a dozen of species across different taxa carry the patronym ehrhardti honouring W. Ehrhardt, but he never published any scientific paper. Ehrhardt also was in contact with the Zoologisches Museum Berlin (ZMB; now part of the Museum für Natur- kunde, Berlin), and, aside from mammal, fish and invertebrate material, he was one of the most important collectors and contributors of herpetological material between the First and the Second World War (Günther 2001). However, major parts of this relatively large herpetological material were, up to now, not determined and therefore not listed in the ZMB catalogue. In the present paper, we have re-examined or determined for the first time all specimens from the Ehrhardt collection. So far, only little information has been published about the life of W. Ehrhardt, apart from his name as collector, but during the preparation of this compilation, we have detected some details on his life in several archives.

The intentions of the present paper are to improve the knowledge on the historical biodiversity and distribution of Brazilian Herpetofauna, and to provide material for future taxonomic revisions.

\footnotetext{
* Corresponding author: e-mail: alexander-gutsche@web.de
} 
Additionally, we give a first biographical overview on the life and work of Wilhelm Ehrhardt.

\section{Biographical sketch of Wilhelm Ehrhardt's life}

Wilhelm Ehrhardt was born on 17 November 1860 in Berbice, British-Guiana (SArchH 18307/1920), now New Amsterdam, Guyana. He most likely descends from German immigrants that settled along the climatic propitious coastline, shortly after this Dutch settlement became a British colony (1831).

It is still unknown, when W. Ehrhardt left British-Guiana, however, on 22 May 1897 he emigrated from Hamburg back to South America on the steamship "Byzanz" together with his first wife Johanna (SArchH VIII A1, Bd. 96). Their destination was the state of Santa Catharina in South Brazil, where several ethnic colonies, e.g. German, Italian, and Belgian, had been established by immigrants. After his arrival at the port of São Francisco do Sul (30 June 1897), Ehrhardt bought land from the 'Hanseatische Kolonisationsgesellschaft mbH' - a German commercial immigration and estate agency and was one of the pioneer settlers at the 'Colonia Hansa Humboldt' (Kormann 1985). Ehrhardt's job history was changeful. He stated 'locksmith' as profession on the immigration list, but, after arriving in Hansa Humboldt (now Corupá), he opened the first general store there. As early as 1897 , he also began a career as animal collector and taxidermist (ZMB S II, Ehrhardt, W., Bl. 15). His background for this profession appears unclear. It seems plausible, that a contact or even kinship to A. Ehrhardt (forename unidentified) - a professional German animal collector in British-Guiana (sic), Surinam, and Barbados around 1860 (ZMB S II, Ehrhardt, A., Bl. 1-3) - may have triggered W. Ehrhardt's professional skills and career in Brazil.

In the following years, Ehrhardt travelled and collected - often accompanied by his second wife Dorothea - intensively across the 'Colonia Hansa Humboldt', which extended roughly from the Rio Negro to the Rio Itapocú in north-southern direction and from Joinville to the Rio Humboldt in east-western direction (fide Ambrosius 1914). Largely on demand of various museums, he collected a wide variety of specimens across vertebrate and invertebrate groups, e.g. fishes, amphibians, reptiles, mammals, arachnids, insects, and annelids. Furthermore, he supplied zoological items such as bird nests, or complete termite nests (up to a weight of $800 \mathrm{~kg}$; Weidner 1967), and also prepared cross-sections of mammal and bird skulls, Didelphis pouches with juveniles, and entrails with parasites. These materials were generally well-labelled, in excellent condition, and the specimens were typically prepared in the near-natural looking 'Ehrhardt style' (Figure 1): "Die Reptilien sind nach dem Leben gespannt, ebenso die Amphibien ..." ['The reptiles as well as the amphibians are mounted in a natural style'] (ZMB S III, Ehrhardt, W., Bl. 31-32).

Because of Ehrhardt's reputation as reliable collector, excellent taxidermist, and inexpensive dealer, he was in contact to numerous museums and scientific institutions, such as the British Museum of Natural History London, the Senckenberg Museum Frankfurt am Main, the Zoologisches Museum München, the Zoologisches Museum Hamburg, the Universität Göttingen, and the Königliche Nervenklinik Tübingen (e.g. NMH DF400/7/20; Panning 1958; SUB Cod. Ms. E. Ehlers 416, Bl. 1). The contacts with the Zoologisches Museum Berlin began in 1907 (ZMB S III, Ehrhardt, W., Bl. 2-3). Initially, caused by a lack of funding, and a Brazilian assortment that already existed in the collections, the purchase of material by the museum was chary. After 1920, between the First and the Second World War, improved financial assets of the museum and personal interventions of the herpetologist Ernst Ahl, made Ehrhardt an important supplier of herpetological material (Günther 2001).

But even before the beginning of the First World War in 1914, Ehrhardt was several times on business travels in Hamburg and other German cities. At the age of 60 (1920), Ehrhardt left Brazil on health reasons, and opened a store for zoological items, beer, wine, tinned food etc. in Hamburg (SArchH 18307/1920). Moreover, between 1923 and 1927 he traded with specimens collected by the Hungary-born Carl Lako in the Amazon basin (ZMB S III, Lako, C., Bl. 4, 9). Only few specimens in the Ehrhardt collections are labelled with 'leg. Lako', but it seems likely that all of the Amazon material was originally collected by Lako, and afterwards prepared and sold by Ehrhardt.

Despite of his heart problems, probably forced by financial worries, Ehrhardt started new collection trips to Santa Catarina in 1927. The following years were bitter for him, often being destitute due to the world economic crisis. Thus, for instance, he had to pay a ship passage to Germany with a debenture bond (ZMB S III Ehrhardt, W., Bl. 107). On 17 February 1933, at the age of 73, he started his last travel from Hamburg to Brazil (SarchH VII A2, Bd. 177).

As yet, date and place of Ehrhardt's death are still unidentified. Based on the last collection dates (1935), and an - apparently posthumous - inquiry of the 'Reichsministerium für Wissenschaft, Erziehung und Volksbildung' ['German ministry for science, education and national education'] at the Zoologisches Museum Berlin about the person W. Ehrhardt (ZMB S III, Ehrhardt, W., Bl. 130), he might have died around 1936 in Brazil.

Ehrhardt's contributions as collector and taxidermist were valuable for zoological science. Inter alia, he supplied the types of several new species, e.g. the torrent frog Hylodes perplicatus (Miranda-Ri- 


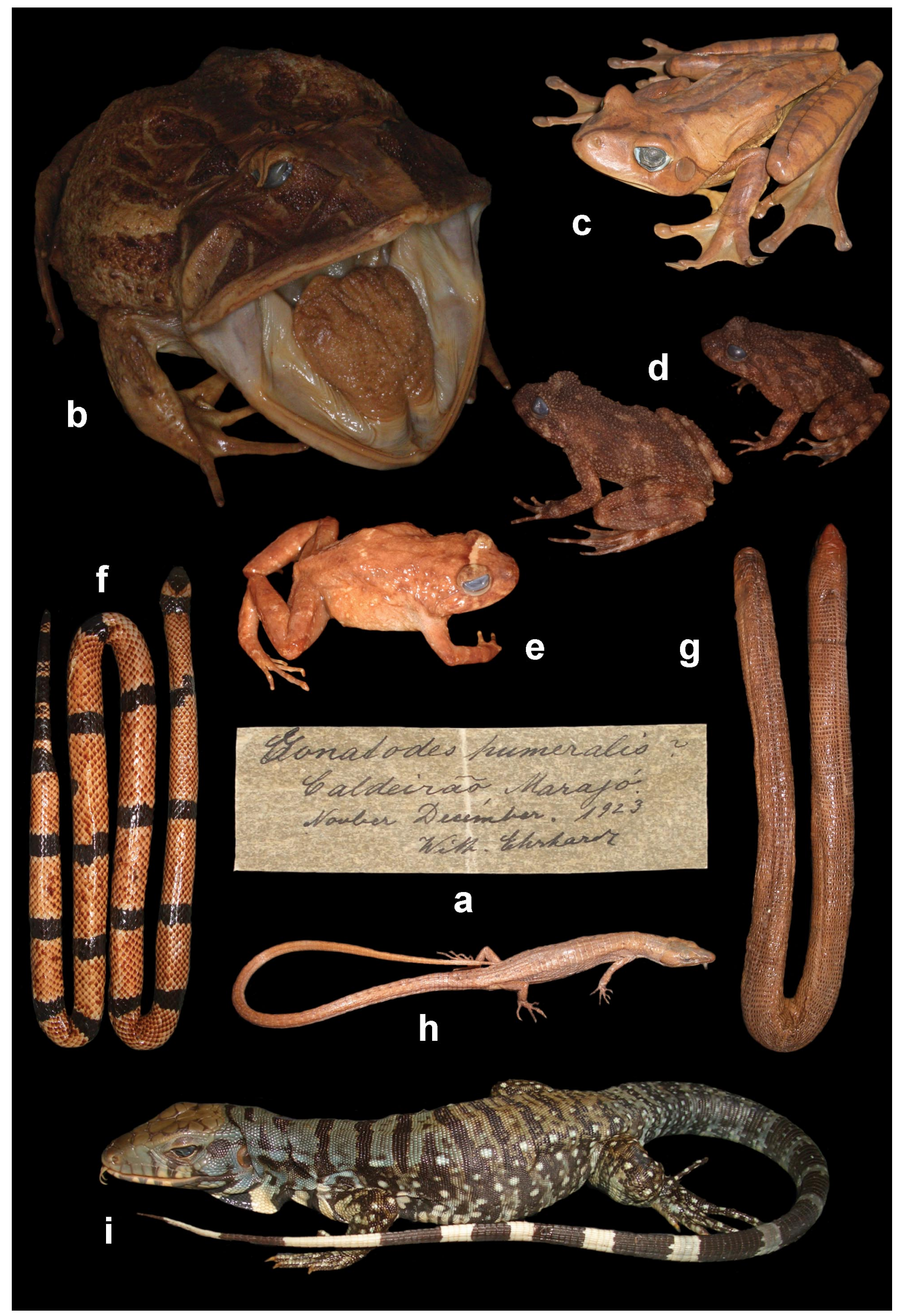


beiro, 1926), and the titi monkey Callicebus purinus Thomas, 1927. In addition, more than a dozen of new species were named erhardti honouring W. Ehrhardt, e.g. the treefrog Aplastodiscus ehrhardti Müller, 1924; the catfish Corydoras ehrhardti Steindachner, 1910; the bat Trachops cirrhosus ehrhardti Felten, 1956; and the bee Lestrimelitta ehrhardti Friese, 1931. Furthermore, a stony votive stele (abolished during the 1940s) was erected at Hansa Humboldt to pay tribute to W. Ehrhardt's social commitments during the early period of the colony (Kormann 1985).

\section{Wilhelm Ehrhardt's Herpetological Collections}

The herpetological collections of W. Ehrhardt in the Museum für Naturkunde, Berlin currently include 2416 specimens representing 22 families with 128 species. The collection represents most of the native Brazilian groups of amphibians and reptiles: Gymnophiona (1 family, 1 species), Anura (5 families, 57 species), Amphisbaenia (1 family, 1 species), Crocodylia (1 family, 2 species), Sauria (7 families, 23 species), Serpentes (4 families, 39 species), Testudines ( 3 families, 5 species). About $70 \%$ of the complete material has been determined and catalogued for the first time during our studies, and all Ehrhardt specimens present in the collection have been re-examined, and if necessary, redetermined. Most of the specimens are stored in ethanol, except noted. Six of the specimens that were once catalogued in the ZMB collection could not be re-located and might have been lost.

The relatively large herpetological collection of W. Ehrhardt contains only one type specimen: Elaps herthae Ahl, 1927: ZMB $30547=$ Atractus latifrons (Günther, 1868). The low number of types and the poor state of determination of the collection might result from the fact that Ernst Ahl, who served as curator at the Zoologisches Museum Berlin between 1921-1941, was notoriously overstrained to determine and catalogue the amount of material arrived during his period of work (Paepke 1995).

The ZMB catalogue also lists 12 specimens of amphibians and reptiles collected by 'Ehrhardt' in Surinam, e.g. the holotype of Hyla conirostris Peters, 1863 (ZMB 4917, probably lost). However, these specimens were sent to the Berlin Museum by the German collector A. Ehrhardt, who was mentioned above. Aside from specimens in the Ehrhardt collection labelled with 'leg. Lako', some specimens from Hansa Humboldt are labelled with 'leg. Globig', which means Ernst Globig, a local snake hunter, who also supplied snakes to the famous Brazilian Butantan Serumtherapy Institute at São Paulo (Jorge Wolfgang Globig, pers. comm. 2006; Kormann 1985).

Both the taxonomy and nomenclature in this catalogue follows the most recent literature available (compare references). We have already adapted the systematic arrangements for hylid frogs by Faivovich et al. (2005) which are mainly accepted, but we did not adapt the new taxonomy of all living amphibians by Frost et al. (2006), however, since this classification is still in discussion. For each specimen the ZMB registration number is provided, along with information derived from the specimen or jar label or the catalogue entries. Localities as given in the specimens list are based on the original names on the labels or in the catalogue. Note that because of some changes in the diction of local names, misspellings, or incorrect names on the labels or catalogue entries, current names and meanings were listed in alphabetical order.

\begin{tabular}{|c|c|}
\hline Ayapua & $\begin{array}{l}\text { Aiapuá, small city near Lago Aiapuá, } \\
\text { State of Amazonas }\end{array}$ \\
\hline Canabonca & $\begin{array}{l}\text { also labelled as Canobonca or Canabou- } \\
\text { ca, maybe local name, State of Amazo- } \\
\text { nas }\end{array}$ \\
\hline Mundurucu & $\begin{array}{l}\text { name for the settlement area of the } \\
\text { Mundurucu Indians, an Amerindian } \\
\text { tribe, that inhabits the area between the } \\
\text { Rios Madeira and Tapajós, about } 200- \\
400 \mathrm{~km} \text { far from the other given locality } \\
\text { Rio Manacapurú }\end{array}$ \\
\hline $\begin{array}{l}\text { Sta. Catharina } \\
\text { Hansa-Humboldt }\end{array}$ & $\begin{array}{l}\text { State of Santa Catarina } \\
\text { the former German 'Colonia Hansa', } \\
\text { and the small city Humboldt, now Muni- } \\
\text { cipality Corupá, State of Santa Catarina }\end{array}$ \\
\hline Rio Humboldt & $\begin{array}{l}\text { tributary that confluences with the Rio } \\
\text { Novo to the Rio Itapocú }\end{array}$ \\
\hline Rio Novo & $\begin{array}{l}\text { tributary that confluences with the Rio } \\
\text { Humboldt to the Rio Itapocú }\end{array}$ \\
\hline Rio Tonantins & $\begin{array}{l}\text { small tributary into the Rio Solimões } \\
\text { (now Rio Amazonas) at the city of To- } \\
\text { nantins }\left(2^{\circ} 46^{\prime} \mathrm{S}, 67^{\circ} 45^{\prime} \mathrm{W}\right) \text {, State of } \\
\text { Amazonas }\end{array}$ \\
\hline Paraná do & $\begin{array}{l}\text { small river (Rio Paraná do Jacaré) and } \\
\text { tributary to Rio Solimões (now Rio } \\
\text { Amazonas), located within the resort } \\
\text { 'Área de Proteção Ambiental de Nha- } \\
\text { mundá', State of Amazonas }\end{array}$ \\
\hline Rio Jutat & $\begin{array}{l}\text { Rio Jutaí, tributary into the Rio Amazo- } \\
\text { nas, State of Amazonas }\end{array}$ \\
\hline Santa Rita & $\begin{array}{l}\text { probably small town at the left margin } \\
\text { of Rio Japurá (Lago Paricá), Município } \\
\text { de Maraã, State of Amazonas }\end{array}$ \\
\hline Kasta & Castaneiro Miri, \\
\hline
\end{tabular}

Fig. 1. Amphibians and reptiles from the Wilhelm Ehrhardt collections in the Museum für Naturkunde, Berlin, showing the typical 'Ehrhardt style' of preparation. a - Original label; b-Ceratophrys aurita (Raddi, 1823); c-Hypsiboas faber (WiedNeuwied, 1821); d - Cycloramphus izecksohni Heyer, 1983; e - Cycloramphus diringshofeni Bokermann, 1957; f - Micrurus corallinus (Merrem, 1820); $\mathbf{g}$ - Leposternon microcephalum Wagler, 1824; h - Placosoma glabella (Peters, 1870); i - Tupinambis merianae (Duméril \& Bibron, 1839). Photos: Axel Kwet (b, d, e), Alexander Gutsche (a, c, f-i; digital processing and graphic design). 


\begin{tabular}{|c|c|}
\hline & $\left(3.3^{\circ} \mathrm{S}, 60.6^{\circ} \mathrm{W}\right)$, State of Amazonas \\
\hline Kaporu & Rio Itapocú, State of Santa Catarina \\
\hline Fonteboa & $\begin{array}{l}\text { Fonte Boa }\left(02^{\circ} 30^{\prime} \mathrm{S}, 66^{\circ} 05^{\prime} \mathrm{W}\right) \text {, State of } \\
\text { Amazonas }\end{array}$ \\
\hline Anatyparana & $\begin{array}{l}\text { Auatí-Paraná, a small river and tribu- } \\
\text { tary to Rio Solimões (now Rio Amazo- } \\
\text { nas), Município de Fonte Boa, State of } \\
\text { Amazonas }\end{array}$ \\
\hline Caldeirão, Marajo & $\begin{array}{l}\text { local name, or incorrect given locality; } \\
\text { Caldeirão is located at Manaus (State } \\
\text { of Amazonas), where most of the Ama- } \\
\text { zonian material was collected, however, } \\
\text { Ehrhardt also mentioned in a letter ma- } \\
\text { terial from the "Amazonasmündungsge- } \\
\text { biet" [mouth of the Amazonas], which } \\
\text { might suggest the Ilha de Marajó }\end{array}$ \\
\hline
\end{tabular}

List of W. Ehrhardt's amphibians and reptiles in the Herpetological Collection

\section{Amphibia}

\section{Gymnophiona}

Caeciliidae

\section{Typhlonectes compressicauda (Duméril \& Bi- bron, 1841)}

ZMB 30991: Ayapua, Rio Purus, Solimões, Amazonas.

Remarks: Specimen could not be located in the collection and is presumably lost.

\section{Anura}

Bufonidae

\section{Bufo abei Baldissera, Caramaschi \& Haddad, 2004}

\section{ZMB 67795: Brasilien;}

ZMB 67803-806, 67812: Sta. Catharina;

ZMB 32013, 67695: Sta. Catharina, Hansa;

ZMB 68079-080: Sta. Catharina, Rio Humboldt-Gebiet;

ZMB 67807-811, 69084-085: Sta. Catharina, Hansa-Humboldt.

Remarks: The Bufo crucifer species group was currently revised, and the specimens presented here are conspecific with the newly described B. abei (Baldissera et al. 2004).

\section{Bufo ictericus Spix, 1824}

ZMB 32000, 55061-068, 68215: Sta. Catharina, Hansa.

\section{Bufo margaritifer-group}

ZMB 30416, 67486-487: Mundurucu, Rio Manacapuru, Amazonas;

ZMB 30979, 67820: Rio Tonantins, Oberer Amazonas;
ZMB 30305, 30316, 30986, 67825-845, 67848, 67877-886: Canobonca, Paraná do Jacaré, Solimões, Amazonas;

ZMB 30302, 67849, 68325: Ayapua, Rio Purus, Solimões, Amazonas.

Remarks: These specimens certainly belong to the B. margaritifer-group, but are difficult to determine. Several authors have indicated that there are a number of sibling species distributed throughout South America and are confused under this name (see additional remarks in Frost 2006).

\section{Bufo marinus (Linnaeus, 1758)}

ZMB 30304, 30315, 67444: Canobonca, Paraná do Jacaré, Solimões, Amazonas.

Centrolenidae

\section{Cochranella oyampiensis (Lescure, 1975)}

ZMB 31079, 55028-030: Manacapuru, Solimões, Amazonas.

Dendrobatidae

Allobates femoralis (Boulenger, 1884)

ZMB 30990: Canobonca, Paraná do Jacaré, Amazonas.

\section{Epipedobates trivittatus (Spix, 1824)}

ZMB 30985, 67850-858: Canobonca, Paraná do Jacaré, Amazonas.

Re marks: ZMB 30985 leg. Carl Lako.

Hylidae

\section{Hylidae}

ZMB 68248-249: Canabonca, Paraná do Jacaré, Solimões, Amazonas.

Remarks: These specimens certainly belong to the family Hylidae, however, a more definite determination was not possible because of their poor state of preservation.

\section{Bokermannohyla hylax (Heyer, 1985)}

ZMB 68260, 68346-357: Sta. Catharina; ZMB 68141: Sta. Catharina, Hansa-Humboldt.

\section{Dendropsophus leucophyllatus (Beireis, 1783)}

ZMB 68246: Canabonca, Paraná do Jacaré, Solimões, Amazonas. 


\section{Dendropsophus microps (Peters, 1872)}

ZMB 68410: Sta. Catharina, Rio Novo-Gebiet.

\section{Dendropsophus minutus (Peters, 1872)}

ZMB 67921, 67950-952: Sta. Catharina, Rio Humboldt-Gebiet.

\section{Dendropsophus nahdereri (Lutz \& Bokermann, 1963)}

ZMB 68318: Sta. Catharina, Rio Humboldt-Gebiet.

\section{Dendropsophus werneri (Cochran, 1952)}

ZMB 67746-777, 68154-155: Sta. Catharina; ZMB 68121-134: Sta. Catharina, Hansa-Humboldt; ZMB 67895-920, 67949: Sta. Catharina, Rio Humboldt-Gebiet;

ZMB 68095: Sta. Catharina, Rio Novo-Gebiet;

ZMB 68118: Sta. Catharina, Rio Novo-Gebiet, Quellfluß des Kaporu.

\section{Hypsiboas albomarginatus (Spix, 1824)}

ZMB 67817: Sta. Catharina, Rio Novo-Gebiet; ZMB 68231: Sta. Catharina, Hansa-Humboldt.

\section{Hypsiboas albopunctatus (Spix, 1824)}

ZMB 68258-259: Brasilien.

\section{Hypsiboas bischoffi (Boulenger, 1887)}

ZMB 67796-797: Brasilien;

ZMB 66957-966, 67798-802, 67943-945, 68156-163, 68358365, 68507-520, 68571-579, 69089: Sta. Catharina; ZMB 32017, 67472-483: Sta. Catharina, Hansa; ZMB 67940, 68222-225, 68230: Sta. Catharina, Hansa-Humboldt; ZMB 67859-876: Sta. Catharina, Quellfluss des Kaporu; ZMB 67946-948, 68396-406: Sta. Catharina, Rio HumboldtGebiet.

\section{Hypsiboas boans (Linnaeus, 1758)}

ZMB 30306: Rio Purus:

ZMB 30981: Ayapua, Rio Purus, Solimões, Amazonas; ZMB 30982: Santa Rita, Rio Solimões, Amazonas.

\section{Hypsiboas faber (Wied-Neuwied, 1821)}

ZMB 31998, 67821-824, 69081-083: Sta. Catharina, Hansa; ZMB 67937-939: Sta. Catharina, Hansa-Humboldt; ZMB 55070-087: Sta. Catharina, Rio Humboldt-Gebiet;
ZMB 68336: Sta. Catharina, Nova Teutônia; ZMB 66821-826: Sta. Catharina, Rio Novo-Gebiet, Quellfluss des Kaporu.

Remarks: The taxidermy of the specimen ZMB 68336 differs clearly from the typical 'Ehrhardt style', and the locality is beyond of Ehrhardt's collection area in Hansa-Humboldt. We therefore assume that this specimen was probably collected and sent to W. Ehrhardt by Fritz Plaumann (1902-1994), a naturalist and entomologist who lived in Nova Teutônia.

\section{Hypsiboas fasciatus (Günther, 1858)}

ZMB 68252: Rio Purus, Amazonas; ZMB 30980: Rio Tonantins, Oberer Amazonas; ZMB 30988, 67780-783: Canobonca, Paraná do Jacaré, Amazonas;

ZMB 68250-251: Canabonca, Paraná do Jacaré, Solimões, Amazonas.

\section{Hypsiboas granosus (Boulenger, 1882)}

ZMB 68326-334: Canabonca, Paraná do Jacaré, Solimões, Amazonas.

\section{Hypsiboas lanciformis Cope, 1871}

ZMB 68247: Canabonca, Paraná do Jacaré, Solimões, Amazonas;

ZMB 68253: Ayapua, Rio Purus, Solimões, Amazonas.

\section{Hypsiboas semilineatus (Spix, 1824)}

ZMB 68206-209, 68254-257: Brasilien; ZMB 66951, 68767-770: Sta. Catharina; ZMB 67484-486, 68135-140, 68227, 68232-238, 68319: Sta. Catharina, Hansa-Humboldt; ZMB 68078, 68407: Sta. Catharina, Rio Humboldt-Gebiet; ZMB 68408-409: Sta. Catharina, Rio Novo-Gebiet, Quellfluß des Kaporu.

\section{Osteocephalus taurinus Steindachner, 1862}

ZMB 29492: Manacapuru, Solimões.

\section{Phyllomedusa bicolor (Boddaert, 1772)}

ZMB 30984: Rio Jutatri, Amazonas; ZMB 30987: Canobonca, Paraná do Jacaré, Amazonas.

\section{Phyllomedusa distincta Lutz, 1950}

ZMB 67791-792: Brasilien;

ZMB 55060, 66871-948, 66974-975, 68273-274: Sta. Catharina; 
ZMB 33602, 67434-443, 68394-395: Sta. Catharina, Hansa; ZMB 67786-788: Rio Novo-Gebiet;

ZMB 55142-152: Sta. Catharina, Rio Humboldt-Gebiet; ZMB 55138-141, 55154-155: Sta. Catharina, Hansa, Rio Humboldt-Gebiet.

\section{Scinax cf. alter (Lutz, 1973)}

ZMB 68265-271, 68494, 68521-525, 68694-752, 68927-957: Sta. Catharina;

ZMB 68176-181, 69023-060: Sta. Catharina, Hansa-Humboldt;

ZMB 68188: Sta. Catharina, Rio Novo-Gebiet.

Remarks: These specimens are allied to $S$. alter, but represent a new (or perhaps two new) species clearly differing in morphology and call characteristics. Various authors agree that there are considerable variation in colouration, size (Lutz 1973; Pombal et al. 1995a) and vocalization (Pombal et al. 1995b) among different populations attributed to S. alter. Additionally, Pombal et al. (1995a) noted, that there may be several species covered by the name S. alter.

\section{Scinax argyreornatus (Miranda-Ribeiro, 1926)}

ZMB 68766: Sta. Catharina.

\section{Scinax fuscovarius (Lutz, 1925)}

ZMB 68495, 68753-760: Sta. Catharina.

\section{Scinax garbei (Miranda-Ribeiro, 1926)}

ZMB 69092: Rio Purus, Valderao, Marajó.

\section{Scinax perereca Pombal, Haddad \& Kasahara, 1995}

ZMB 68262-264, 68311-312, 68493, 68580-634, 68812-926, 68969-69022: Sta. Catharina; ZMB 68142, 69061-080: Sta. Catharina, Hansa-Humboldt; ZMB 68190: Sta. Catharina, Rio Novo-Gebiet; ZMB 67980-68059: Sta. Catharina, Hansa, Rio HumboldtGebiet;

ZMB 69093-095: Sta. Catharina, Hansa, Rio Novo-Gebiet.

\section{Scinax perpusillus (Lutz \& Lutz, 1939)}

ZMB 67794: Brasilien;

ZMB 68444-445: Sta. Catharina, Hansa-Humboldt.

\section{Scinax rizibilis (Bokermann, 1964)}

ZMB 68526-570, 68635-693，68790-811，68958-968: Sta. Catharina;
ZMB 68239: Sta. Catharina, Hansa-Humboldt; ZMB 55153: Sta. Catharina, Rio Humboldt-Gebiet; ZMB 67953-979: Sta. Catharina, Hansa, Rio Humboldt-Gebiet.

\section{Scinax ruber (Laurenti, 1768)}

ZMB 30983: Fonteboa, Rio Solimões, Amazonas; ZMB 31081, 67460-471: Manacapuru, Solimões, Amazonas; ZMB 68337: Ayapua, Rio Purus, Solimões, Amazonas.

\section{Trachycephalus mesophaeus (Hensel, 1867)}

ZMB 32007, 64971-65073, 67446-459, 68243-244: Sta. Catharina, Hansa;

ZMB 68228-229: Sta. Catharina, Hansa, Quellfluß des Kaporu.

\section{Trachycephalus venulosus (Laurenti, 1768)}

ZMB 31080: Manacapuru, Solimões, Amazonas; ZMB 30303, 30307, 68321-323: Ayapua, Rio Purus, Solimões, Amazonas.

Leptodactylidae

\section{Adenomera nana (Müller, 1922)}

ZMB 66829-844, 66950, 66976-67011, 67814: Sta. Catharina; ZMB 67815-816: Rio Novo-Gebiet.

Remarks: Up to date, this taxon was treated as a junior synonym of $A$. marmorata (see Frost 2006), but based on bioacoustic studies, it was recently suggested to revalidate the species status (Kwet in press).

\section{Ceratophrys aurita (Raddi, 1823)}

ZMB 61806, 67245: Sta. Catharina; ZMB 31997, 54316-327: Sta. Catharina, Hansa; ZMB 38356, 67288-289, 68216-221: Sta. Catharina, HansaHumboldt.

\section{Crossodactylus dispar Lutz, 1925}

ZMB 68168, 68170-176, 68276-308, 68386-393, 68446-492, 68765: Sta. Catharina;

ZMB 32011-012, 67487-694: Sta. Catharina, Hansa; ZMB 68421-426: Sta. Catharina, Hansa-Humboldt; ZMB 68146-153: Sta. Catharina, Rio Novo; ZMB 68100-117: Sta. Catharina, Rio Novo-Gebiet; ZMB 67922-934: Sta. Catharina, Rio Novo-Gebiet, Quellfluß des Kaporu;

ZMB 68074-077: Sta. Catharina, Rio Humboldt-Gebiet.

Remarks: The taxonomy of Crossodactylus is unresolved and the species groups are discussed (Bastos \& Pombal 1995). However, other specimens 
collected by W. Ehrhardt at the same locality were identified by Cochran (1955) as Crossodactylus dispar, and until a more precise review, this is the name available for this population.

\section{Cycloramphus cf. asper Werner, 1899}

ZMB 67942, 68499; 68776-789: Sta. Catharina; ZMB 32014, 68226: Sta. Catharina, Hansa.

Remarks: The taxonomy of the genus Cycloramphus is difficult. Eight closely related species of $C y$ cloramphus are known from the State of Santa Catarina (C. asper, C. bolitoglossus, C. catarinensis, C. cedrensis, C. diringshofeni, C. izecksohni, C. rhyakonastes, and C.valae). Off these, four are with reduced to considerable webbing (see Heyer 1983a, b). The specimens here analysed display considerable webbing, and therefore are tentatively referred as Cycloramphus cf. asper.

\section{Cycloramphus bolitoglossus (Werner, 1897)}

ZMB 29997: Rio Novo-Gebiet;

ZMB 68240: Sta. Catharina, Hansa;

ZMB 68184-186: Sta. Catharina, Rio Humboldt-Gebiet.

\section{Cycloramphus diringshofeni Bokermann, 1957}

ZMB 68191: Sta. Catharina, Rio Novo-Gebiet.

\section{Cycloramphus izecksohni Heyer, 1983}

ZMB 68496-498, 68771-775: Sta. Catharina; ZMB 32019, 67225-227: Sta. Catharina, Hansa; ZMB 67935-936: Sta. Catharina, Rio Novo-Gebiet; ZMB 68081-087: Sta. Catharina, Rio Humboldt-Gebiet; ZMB 68119-120: St. Catharina, Rio Novo-Gebiet, Quellfluss des Kaporu;

ZMB 68192-195: Sta. Catharina, Hansa, Rio Humboldt-Gebiet.

\section{Eleutherodactylus binotatus (Spix, 1824)}

ZMB 67941, 68261: Sta. Catharina; ZMB 67290-292: Sta. Catharina, Hansa; ZMB 69090-091: Joinville [Santa Catarina State], Südamerika.

\section{Eleutherodactylus cf. guentheri (Steindachner, 1864)}

ZMB 67793: Brasilien; ZMB 66955-956: Rio Novo-Gebiet; ZMB 67813, 68182-183: Sta. Catharina, Rio Humboldt-Gebiet.
Remarks: These specimens may belong to E. guentheri, although this species is stated to be morphologically very similar to its cryptic sibling species E. henselii (Peters, 1870) (Kwet \& Solé 2005). Both E. guentheri and E. henselii are distributed in northeastern Santa Catarina and bioacoustic data are necessary to take a final decision about their species identity.

\section{Flectonotus cf. fissilis (Miranda-Ribeiro, 1920)}

ZMB 68189: Sta. Catharina, Rio Novo-Gebiet.

Remarks: This specimen is allied to F. fissilis. According to Duellman and Gray (1983), F. fissilis inhabits the humid forests in the mountains of southeastern Brazil - Serra da Bocaina, Serra do Mar, and Serra dos Órgãos in the states of São Paulo, Rio de Janeiro, and Espírito Santo. The specimen may represent a new species rather than reflect an increasing geographic distribution range of $F$. fissilis, but more samples are necessary for a precise identification.

\section{Hylodes perplicatus (Miranda-Ribeiro, 1926)}

ZMB 68164-167, 68169, 68313-317, 68366-385, 68500-505: Sta. Catharina;

ZMB 67421-433: Sta. Catharina, Hansa;

ZMB 68427-443: Sta. Catharina, Hansa-Humboldt;

ZMB 68060-073: Sta. Catharina, Rio Humboldt-Gebiet;

ZMB 67887-894: Sta. Catharina, Rio Novo-Gebiet;

ZMB 68096-098: Sta. Catharina, Rio Novo;

ZMB 68143-145: Sta. Catharina, Rio Novo-Gebiet, Quellfluß des Kaporu.

\section{Leptodactylus notoaktites Heyer, 1978}

ZMB 68310, 68761-764: Sta. Catharina;

ZMB 32005, 67293-295, 67818-819, 68241-242: Sta. Catharina, Hansa;

ZMB 68099: Sta. Catharina, Rio Novo-Gebiet;

ZMB 29482: Sta. Catharina, Humboldt, Gebiet des Rio Novo.

\section{Leptodactylus ocellatus (Linnaeus, 1758)}

ZMB 67012-014: Brasilien; ZMB 66827-828: Sta. Catharina; ZMB 31999, 67228-230: Sta. Catharina, Hansa; ZMB 31082, 67445: Manacapuru, Solimões, Amazonas; ZMB 68338-340: Ayapua, Rio Purus, Solimões, Amazonas; ZMB 68341-344: Rio Purus, Solimões, Amazonas.

\section{Leptodactylus pentadactylus (Laurenti, 1768)}

ZMB 30972: Rio Jutatri, Amazonas; ZMB 68335: Lago do Jaraci, Rio Negro, Amazonas. 


\section{Leptodactylus petersii (Steindachner, 1864)}

ZMB 31083, 54356-378: Manacapuru, Solimões, Amazonas.

\section{Lithodytes lineatus (Schneider, 1799)}

ZMB 68324: Mundurucu, Rio Manacapuru, Amazonas.

\section{Physalaemus cuvieri Fitzinger, 1826}

ZMB 67784-785, 68275: Sta. Catharina;

ZMB 68088-094, 68187: Sta. Catharina, Rio Humboldt-Gebiet.

\section{Physalaemus nanus (Boulenger, 1888)}

ZMB 68309: Sta. Catharina.

\section{Physalaemus olfersii (Lichtenstein \& Martens, 1856)}

ZMB 67789-790: Rio Novo-Gebiet.

\section{Proceratophrys boiei (Wied-Neuwied, 1824)}

ZMB 66845-848, 67232-238, 68320, 69088: Sta. Catharina; ZMB 32003, 32006, 32016, 32018, 48714, 67239-244: Sta. Catharina, Hansa;

ZMB 55088-090: Rio Novo-Gebiet;

ZMB 55091-137: Sta. Catharina, Hansa, Rio Humboldt-Gebiet.

Proceratophrys subguttata Izecksohn, Cruz \& Peixoto, 1999 "1998”

ZMB 67275: no locality data given; ZMB 32015: Sta. Catharina, Hansa.

Scythrophrys sawayae (Cochran, 1953)

ZMB 68272: Sta. Catharina, Hansa, Rio Humboldt-Gebiet.

\section{Reptilia}

\section{Testudines}

Chelidae

\section{Chelus fimbriatus (Schneider, 1783)}

ZMB 30121: Solimões;

ZMB 30973: Rio Jutatri, Amazonas.
Remarks: ZMB 30121 could not be located in the collection and is presumably lost. ZMB 30973 leg. Carl Lako.

\section{Hydromedusa tectifera Cope, 1869}

ZMB 31996, 36217: Sta. Catharina, Hansa.

\section{Phrynops nasutus (Schweigger, 1812)}

ZMB 30936: Santa Rita, Rio Solimões, Amaz.[onas].

Remarks: The specimen could not be located in the collection and is presumably lost.

Podocnemididae

\section{Podocnemis expansa (Schweigger, 1812)}

ZMB 55159: Ayapua, Rio Purus, Solimões, Amazonas.

Remarks: The specimen is a dried skull.

Testudinidae

\section{Geochelone denticulata (Linnaeus, 1766)}

ZMB 30938: Santa Rita, Rio Solimões, Amazonas.

Rem arks: ZMB 30938 leg. Carl Lako.

\section{Amphisbaenia}

Amphisbaenidae

Leposternon microcephalum Wagler, 1824

ZMB 68345: Sta. Catharina; ZMB 31987, 69768-770, 32010, 69811: Sta. Catharina, Hansa; ZMB 64405-420: Sta. Catharina, Hansa-Humboldt.

Remarks: The specimens ZMB 32010 and 69811 are juveniles, the specimen ZMB 68345 is a egg including an embryo.

\section{Sauria}

\section{Gekkonidae}

\section{Gonatodes humeralis (Guichenot, 1855)}

ZMB 30317, 69738-750: Manacapuru, Solimões, Amazonas; ZMB 31073, 31076, 69721-737, 69738-750: Caldeirão, Marajó, Amazonas. 
Hemidactylus mabouia (Moreau de Jonnès, Norops trachyderma (Cope, 1876) 1818)

ZMB 69833-834: Sta. Catharina;

ZMB 69835: Rio Novo-Gebiet.

ZMB 30961: Santa Rita, Rio Solimões, Amaz.[onas].

Scincidae

Gymnophthalmidae

Mabuya bistriata (Spix, 1825)

Cercosaura schreibersii schreibersii Wiegmann, ZMB 69837: Ayapua, Rio Purus, Solimões, Amazonas. 1834

ZMB 69886: Sta. Catharina, Hansa.

Placosoma glabella (Peters, 1870)

ZMB 69888: Sta. Catharina;

ZMB 57005: Sta. Catharina, Humboldt.

Leiosauridae

Mabuya nigropunctata (Spix, 1825)

ZMB 29354, 29357: Caldeirão, Marajó.

Teiidae

Ameiva ameiva (Linnaeus, 1758)

ZMB 29359, 31077: Manacapuru, Amazonas.

\section{Enyalius iheringii Boulenger, 1885}

ZMB 36942: Sta. Catharina;

ZMB 31995, 69845: Sta. Catharina, Hansa;

ZMB 36866, 37135, 37137, 38559, 56022-025, 69839-844,

69871-885: Sta. Catharina, Hansa-Humboldt.

Polychrotidae

Anolis punctatus Daudin, 1802

ZMB 30962: Santa Rita, Rio Solimões, Amaz.[onas].

\section{Anolis transversalis Duméril, 1851}

ZMB 30964: Santa Rita, Rio Solimões, Amaz.[onas].

\section{Norops fuscoauratus (D'Orbigny in Duméril \& Bibron, 1837)}

ZMB 30963, 69687-689: Santa Rita, Rio Solimões, Amaz. [onas].

\section{Crocodilurus amazonicus Spix, 1825}

ZMB 29355: Manacapuru, Amazonas;

ZMB 30295: Canabonca, Paraná do Jacaré, mittlerer Amazonas;

ZMB 30300: Rio Purus;

ZMB 30978: Ayapua, Rio Purus, Amazonas.

Re marks: ZMB 30978 leg. Carl Lako.

\section{Kentropyx altamazonica (Cope, 1876)}

ZMB 69836, 69838: Ayapua, Rio Purus, Solimões, Amazonas.

\section{Kentropyx calcarata Spix, 1825}

ZMB 29356: Caldeirão, Marajó.

\section{Tupinambis merianae (Duméril \& Bibron, 1839)}

ZMB 32001, 69823-69829: Sta. Catharina, Hansa-Humboldt. Remarks: The specimens ZMB 69823-825 are

\section{Norops nitens (Wagler, 1830)}

ZMB 69887: Mundurucu, Rio Manacapuru, Amaz.[onas]. freshly hatched juveniles, the specimens ZMB 69826-828 are juveniles in the process of hatching.

Tropiduridae

Norops ortonii (Cope, 1868)

ZMB 30977: Rio Tonantins, Oberer Amazonas; 


\section{Plica umbra ochrocollaris (Spix, 1825)}

ZMB 30975, 52303: Rio Tonantins, Oberer Amazonas; ZMB 36945: Canabonca, Paraná do Jacaré, Solimões, Amazonas.

Uracentron azureum guentheri Boulenger, 1894

ZMB 30974: Rio Jutatri, Amazonas.

\section{Uracentron flaviceps (Guichenot, 1855)}

ZMB 30973: Rio Jutatri, Amazonas.

\section{Uranoscodon superciliosus (Linnaeus, 1758)}

ZMB 29358: Manacapuru, Amazonas;

ZMB 30296, 30310: Lago de Arara, Solimões, Amazonas; ZMB 30301, 30313-314: Canabonca, Paraná do Jacaré, Solimões, Amazonas.

\section{Serpentes}

Colubridae

\section{Colubridae}

ZMB 31968: Sta. Catharina, Hansa.

Remarks: The specimen was catalogued only with the family name, however, it could not be located in the collection and is presumably lost.

\section{Atractus latifrons (Günther, 1868)}

ZMB 30547: Mundurucu, Rio Manacapuru, Amazonas.

Remarks: Holotype of Elaps herthae Ahl, 1927, synonymized by Peters \& Orejas-Miranda (1970).

\section{Atractus snethlageae Cunha \& Nascimento, 1983}

ZMB 47780: Lago de Arara, Solimões, Amazonas.

\section{Chironius exoletus (Linnaeus, 1758)}

ZMB 30992, 66663: Canabonca, Paraná do Jacaré, Amazonas;

ZMB 47704: Sta. Catharina, Hansa;

ZMB 66587: Sta. Catharina, Hansa-Humboldt

Re ma rks: ZMB 30992, 66663 leg. Carl Lako.
Clelia plumbea (Wied-Neuwied, 1820)

ZMB 69272-273: Sta. Catharina, Hansa-Humboldt; ZMB 69274: Sta. Catharina, Hansa.

\section{Dipsas albifrons (Sauvage, 1884)}

ZMB 69269-270: Sta. Catharina, Hansa; ZMB 47702, 54168: Sta. Catharina, Hansa-Humboldt.

\section{Dipsas alternans (Fischer, 1885)}

ZMB 69266: Sta. Catharina, Hansa-Humboldt.

\section{Dipsas catesbyi (Sentzen, 1796)}

ZMB 69271: Kastaneiro Miri, Solimões, Amazonas

\section{Dipsas indica indica Laurenti, 1768}

ZMB 47740: Ayapua, Rio Purus, Solimões, Amazonas.

\section{Dipsas neivai Amaral, 1926}

ZMB 69267-268: Sta. Catharina, Hansa-Humboldt

\section{Echinanthera undulata (Wied-Neuwied, 1824)}

ZMB 47782, 49174: Sta. Catharina, Hansa; ZMB 69258-261: Sta. Catharina, Hansa-Humboldt.

\section{Helicops polylepis Günther, 1861}

ZMB 30968: Santa Rita, Rio Solimões, Amaz.[onas]; ZMB 30993: Canabonca, Paraná do Jacaré, Amazonas.

Remarks: ZMB 30968 is on loan to Rubens Nobuo Yuki (Brazil) since the 21 January 1997, therefore, species determination could not be proofed.

\section{Helicops hagmanni Roux, 1910}

ZMB 30970: Rio Tonantins, Oberer Amazonas.

Remarks: The specimen is on loan to Rubens Nobuo Yuki (Brazil) since the 21 January 1997, therefore, species determination could not be proofed.

Hydrodynastes gigas (Duméril, Bibron \& Duméril, 1854)

ZMB 47680: Ayapua, Rio Purus, Solimões, Amazonas. 


\section{Imantodes cenchoa cenchoa (Linnaeus, 1758)}

ZMB 47739: Kastaneiro Miri, Solimões, Amazonas; ZMB 64727: Caldeirão, Marajó, Amazonas-Gebiet.

\section{Leptodeira annulata annulata (Linnaeus, 1758)}

ZMB 30994: Canabonca, Paraná do Jacaré, Amazonas; ZMB 47824: Ayapua, Rio Purus, Solimões, Amazonas.

Remarks: ZMB 30994 leg. Carl Lako.

\section{Leptophis ahaetulla nigromarginatus (Günther, 1866)}

ZMB 30969: Santa Rita, Rio Solimões, Amaz.[onas].

Re marks: ZMB 30969 leg. Carl Lako.

\section{Leptophis ahaetulla ortonii Cope, 1876}

ZMB 47743: Lago de Arara, Solimões, Amazonas;

ZMB 47756: Ayapua, Rio Purus, Solimões, Amazonas.

\section{Liophis miliaris orinus (Griffin, 1916)}

ZMB 69262: Sta. Catharina;

ZMB 31989, 32002, 32004, 47785, 47768, 69256: Sta. Catharina, Hansa;

ZMB 67072: Sta. Catharina, Hansa-Humboldt.

\section{Liophis reginae semilineatus (Wagler, 1824)}

ZMB 30971: Santa Rita, Rio Solimões, Amaz.[onas].

Remarks: ZMB 30971 leg. Carl Lako.

\section{Oxybelis aeneus (Wagler, 1824)}

ZMB 30311: Caldeirão, Marajó.

\section{Oxybelis fulgidus (Daudin, 1803)}

ZMB 30966: Santa Rita, Rio Solimões, Amaz.[onas]; ZMB 56830: Rio Negro bei Manaus, Amazonas; ZMB 62514: Rio Purus, Amazonas.

Remarks: The specimen ZMB 62514 is a head and neck; ZMB 30966 leg. Carl Lako.

Oxyrhopus clathratus Duméril, Bibron \& Duméril, 1854

ZMB 31992: Sta. Catharina, Hansa;

ZMB 65418-420, 65471-477: Sta. Catharina, Hansa-Humboldt.
Philodryas aestiva (Duméril, Bibron \& Duméril, 1854)

ZMB 69257: Sta. Catharina, Hansa, Brasil.

Sibynomorphus neuwiedi (Ihering, 1911)

ZMB 69265: Sta. Catharina, Hansa-Humboldt.

Spilotes pullatus (Linnaeus, 1758)

ZMB 47779: Sta. Catharina, Hansa.

Thamnodynastes sp. 1 (fide Franco \& Ferreira, 2002)

ZMB 69263: Sta. Catharina, Hansa-Humboldt.

Re marks: The Brazilian species are currently under revision, and the specimen analysed here is identical with an undescribed species, the characters of which are given in Franco \& Ferreira (2002).

\section{Tropidodryas serra (Schlegel, 1837)}

ZMB 47774, 47784, 65483-484: Sta. Catharina, Hansa-Humboldt.

\section{Tropidodryas striaticeps (Cope, 1870 “1869”)}

ZMB 47772, 47775, 47783: Sta. Catharina, Hansa; ZMB 65485-486: Sta. Catharina, Hansa-Humboldt.

\section{Xenodon neuwiedii Günther, 1863}

ZMB 66758-760, 66805-807: Sta. Catharina; ZMB 31994, 47713，47766, 47773, 47832: Sta. Catharina, Hansa;

ZMB 47767, 64646-656: Sta. Catharina, Hansa-Humboldt.

\section{Xenoxybelis argenteus (Daudin, 1803)}

ZMB 63774: Lago de Arara, Solimões, Amazonas.

Elapidae

\section{Elaps sp.}

ZMB 31988: Sta. Catharina, Hansa.

Remarks: The South American members of the genus Elaps now belong to the genus Micrurus, however, this specimen could not be located in 
the collection for determination and is presumably lost.

\section{Micrurus corallinus (Merrem, 1820)}

ZMB 59093-095: Sta. Catharina;

ZMB 31918, 35743, 69246-248, 69253: Sta. Catharina, Hansa; ZMB 47787-788, 47790, 59096-105, 69249-252, 69254: Sta. Catharina, Hansa-Humboldt.

Remarks: ZMB 69254 leg. Globig.

Typhlopidae

\section{Typhlops reticulatus (Linnaeus, 1758)}

ZMB 30960: Santa Rita, Rio Solimões, Amaz.[onas].

\section{Viperidae}

\section{Bothrops sp.}

ZMB 32008-009: Sta. Catharina, Hansa.

Remarks: The specimens are embryos, therefore, a more detailed species determination was not possible.

\section{Bothrops atrox (Linnaeus, 1758)}

ZMB 30965: Anatyparana, Amazonas; ZMB 30967: Rio Tonantins, Amazonas; ZMB 48649: Rio Manacapuru, Amazonas.

\section{Bothrops jararaca (Wied-Neuwied, 1824)}

ZMB 69255: Sta. Catharina;

ZMB 45807-816, 47781, 47849, 65502，65505，65509-522, 65497-500: Sta. Catharina, Hansa;

ZMB 45803-806, 65501, 65503-504: Sta. Catharina, HansaHumboldt.

Remarks: ZMB 65501, 65503 leg. Globig.

\section{Bothrops jararacussu Lacerda, 1884}

ZMB 31990-991，36261，37227，47769，47776-778，47786, 65523: Sta. Catharina, Hansa;

ZMB 63585-589, 65508: Sta. Catharina, Hansa-Humboldt.

\section{Crotalus durissus (Linnaeus, 1758)}

ZMB 47796: Brasilien.

\section{Crocodylia}

Crocodylidae

\section{Caiman sp.}

ZMB 30662: Rio Purus.

Remarks: The specimen could not be located in the collection and is presumably lost.

\section{Melanosuchus niger (Spix, 1825)}

ZMB 36591: Rio Manacapuru, Amazonas.

\section{Appendix}

Specimens without data, but showing the typical 'Ehrhardt style' of preparation, and therefore presumably collected by W. Ehrhardt.

Colubridae

Sibynomorphus neuwiedi (Ihering, 1911)

ZMB 69264: no locality data given.

Viperidae

Bothrops jararacussu Lacerda, 1884

ZMB 47791, 63517-518: no locality data given.

\section{Acknowledgements}

We would like to thank Hannelore Landsberg from the 'Historische Bild- und Schriftgutsammlung' of the Museum für Naturkunde, Berlin, the Hamburg State Archive, and the Joinville State Archive, Brazil, for access to their resources. We are indebted to the city hall of Corupá, especially to Ernesto Felipe Blunk and Lane A. K. Weidner for providing literature, and to Jorge Wolfgang Globig, Brazil, for helpful information. Finally, we are indebted to O. Behrend for editorial work on the English text.

\section{References}

Ambrosius, E. (Hrsg.). 1914. Andrees Allgemeiner Handatlas. 6. Auflage. - Verlag von Velhagen und Klasing, Bielefeld und Leipzig.

Baldissera Jr., F. A., Caramaschi, U. \& Haddad, C. F. B. 2004. Review of the Bufo crucifer species group, with descriptions of two new related species (Amphibia, Anura, Bufonidae). - Arquivos do Museo Nacional, Rio de Janeiro 62: 255-282. 
Bastos, R. P. \& Pombal Jr., J. P. 1995. New species of Crossodactylus (Anura: Leptodactylidae) from the Atlantic rain forest of southeastern Brazil. - Copeia 1995: 436-439.

Cochran, D. M. 1955. Frogs of south-eastern Brazil. - United States National Museum Bulletin 206: 1-423.

Duellman, W. E. \& Gray, P. 1983. Developmental biology and systematics of the egg-brooding hylid frogs, genera Flectonotus and Fritziana. - Herpetologica 39: 333-359.

Faivovich, J., Haddad, C. F. B., Garcia, P. C. A., Frost, D. R., Campbell, J. A. \& Wheeler, W. C. 2005. Systematic review of the frog family Hylidae, with special reference to Hylinae: phylogenetic analyses and taxonomic revision. - Bulletin of the American Museum of Natural History, New York 294: 1-240.

Franco, F. L. \& Ferriera, T. G. 2002. Descricão de uma nova especies de Thamnodynastes Wagler, 1830 (Serpentes, Colubridae) do nordeste brasileiro, com comentarios sobre o genero. - Phyllomedusa 1: 57-74.

Frost, D. R. 2006. Amphibian species of the World: an Online Reference. Version 4.0 (20 August, 2006). - http:// research.amnh.org/herpetology/amphibia/index.php. American Museum of Natural History, NY, USA.

Frost, D. R., Grant, T., Faivovich, J., Bain, R. H., Haas, A., Haddad, C. F. B., De Sá, R. O., Channing, A., Wilkinson, M., Donnellan, S. C., Raxworthy, Ch. J., Campbell, J. A., Blotto, B. L., Moler, P., Drewes, R. C., Nussbaum, R. A., Lynch, J. D., Green, D. M. \& Wheeler, W. C. 2006. The amphibian tree of life. - Bulletin of the American Museum of Natural History 297: 1-370.

Günther, R. 2001. Zur Geschichte der herpetologischen Sammlung des Museums für Naturkunde Berlin. In Rieck, W., Hallmann, G. \& Bischoff, W. (Hrsg.). Die Geschichte der Herpetologie und Terrarienkunde im deutschsprachigen Raum: pp. 359-374. - Mertensiella 12.

Heyer, W. R. 1983a. Notes on the frog genus Cycloramphus (Amphibia: Leptodactylidae), with descriptions of two new species. - Proceedings of the Biological Society of Washington 96: 548-559.

- 1983b. Variation and systematics of frogs of the genus $C y$ cloramphus (Amphibia, Leptodactylidae). - Arquivos de Zoologia 30: 235-339.

Kormann, J. 1985. Hansa Humboldt ontem, hoje Corupá. Florianópolis, Brazil.

Kwet, A. in press. Bioacoustics in the genus Adenomera (Anura: Leptodactylidae) from Santa Catarina southern Brazil. - Abstracts of the $13^{\text {th }}$ ordinary general meeting of the Societas Europaea Herpetologica (SEH), Bonn, 27. September - 02. Oktober 2005.

Kwet, A. \& Solé, M. 2005. Validation of Hylodes henselii Peters, 1870, from Southern Brazil and Description of Acoustic Variation in Eleutherodactylus guentheri (Anura: Leptodactylidae). - Journal of Herpetology 39: 521-532.

Lutz, B. 1973. Brazilian species of Hyla. - Austin \& London, University of Texas Press.

NMH (Natural History Museum, London). - Ehrhardt, Wilhelm (Brazil), DF400/7/20, 1912.

Paepke, H.-J. 1995. Über das Leben und Werk von Ernst Ahl. - Mitteilungen des Zoologischen Museums Berlin 71: 79-101.

Panning, A. 1958. Beiträge zur Geschichte des Zoologischen Staatsinstituts und Zoologischen Museums in Hamburg. 4. Teil. Die Förderung des Museums durch Hamburgs Linienreederei. - Mitteilungen aus dem Hamburgischen Zoologischen Museum und Institut 56: 1-30.

Peters, J. A. \& Orejas-Miranda, B. 1970. Catalogue of the neotropical squamata: Part I. Snakes. - United States National Museum Bulletin, Smithsonian Institution Press, Washington 297: 1-347.

Pombal Jr., J. P., Haddad, C. F. B. \& Kasahara, S. 1995a. A new species of Scinax (Anura: Hylidae) from southeastern Brazil, with comments on the genus. - Journal of Herpetology 29: 1-6.

Pombal Jr., J. P., Bastos, R. P. \& Haddad, C. F. B. 1995b. Vocalizações de algumas espécies do gênero Scinax (Anura, Hylidae) do sudeste do Brasil e comentários taxonômicos. - Naturalia 20: 213-225.

SArchH (Staatsarchiv Hamburg). - Gewerbeanmeldeschein Nr. 18307/1920, Karteiregister Nr. 1, 30. 11. 1920; Auswandererlisten, VIII A1, Band 96, 22. 05. 1897; VII A2, Band 177, 17. 02. 1933 .

SUB (Niedersächsische Staats- und Universitätsbibliothek Göttingen). - W. Ehrhardt an E. Ehlers, 12. 03. 1914; Cod. Ms. E. Ehlers 416, Blatt 1.

Weidner, H. 1967. Geschichte der Entomologie in Hamburg. - Abhandlungen und Verhandlungen des Naturwissenschaftlichen Vereins in Hamburg, N. F., Supplement 9: 211-212.

ZMB (Zoologisches Museum Berlin). - Museum für Naturkunde der Humboldt-Universität zu Berlin, Historische Bild- und Schriftgutsammlung, S II, Ehrhardt, W., Blatt 15; S III, Ehrhardt, W., Blatt 2-3, 31-32, 107, 130; S II, Ehrhardt, A., Blatt 1-3; S III, Lako, C., Blatt 4, 9. 